der Aufbau für $100 \mathrm{~kg} / \mathrm{d}$ im Wesentlichen aus einem modular aufgebauten Mikroreaktor für den ersten Teil der Reaktion, der mit einer starken Wärmefreisetzung verbunden ist, gefolgt von einem 20fach-Rohrbündelreaktor mit Strukturgrößen im Bereich einiger Millimeter zur Vervollständigung der Reaktion, die nur noch $\mathrm{zu}$ einer geringen Wärmefreisetzung führt. Die Reaktoraufbauten gestatten die Überführung des derzeitigen konventionellen BatchProzesses mit seinen Limitierungen in einen kontinuierlichen, intensivierten Prozess - verbunden mit einer deutlich reduzierten Menge an karzinogenem Diethylsulfat im Reaktor. Fokus dieses Beitrags ist die Beschreibung der Entwicklung des Reaktorkonzeptes für den Produktionsmaßstab ausgehend von den ersten kontinuierlichen Laborversuchen. Damit wird der verfolgte Ansatz für das Scale-up eines multi-skaligen Reaktoraufbaus beispielhaft verdeutlich. Ergänzend werden auch die Ergebnisse der nicht-reaktiven Charakterisierung der realisierten Reaktoraufbauten bezüglich Druckabfall, Wärmetausch und Verweilzeitverhalten zur experimentellen Validierung der während des Scaleups gemachten Annahmen dargestellt.

\title{
P3.18
}

\section{Vielfalt und Vielseitigkeit von Trommelfiltern}

Dr.-Ing. R. Bott ${ }^{1)}$ (E-Mail: bottrb@aol.com), Dr.-Ing. T. Langeloh ${ }^{1)}$

${ }^{1)}$ BOKELA GmbH, Tullastraße 64, D-76131 Karlsruhe, Germany

DOI: $10.1002 /$ cite. 200950620

Trommelfilter sind die am weitesten verbreitete Bauart der kontinuierlichen Drehfilter. Kein anderes Drehfilter wird in so vielen Anwendungsbereichen für die Filtration, Waschung, Pressung und Entfeuchtung so vieler unterschiedlicher Produkte eingesetzt wie das Trommelfilter. Ein zentrales Einsatzgebiet sind Filtrationsaufgabenstellungen, bei denen der Filterkuchen gewaschen werden muss. Im Vergleich zu Bandfiltern, den typischen Waschfiltern, beanspruchen Trommelfilter eine deutlich geringere Stellfläche und bieten zudem eine unschlagbare Vielfalt an Möglichkeiten, um unterschiedlichste Filterkuchen vom Filtertuch abzunehmen, wie z. B durch:

- Druckluftrückstoß und Schaber für relativ trockene, krümelige Kuchen
- Abnahmewalze für sehr feinkörnige, dünne, klebrige Filterkuchen

- ablaufendes Filtertuch, wenn eine intensive Tuchwaschung notwendig ist

- ablaufende Schnüre oder Ketten für dicke oder vliesähnliche Filterkuchen

- Precoatschicht mit Abnahmemesser für sehr dünne Kuchen (0,1-1 mm) Eine neue Generation von Trommelfiltern mit fortschrittlichem Design bringt nun die Vorzüge und Vielseitigkeit der Trommelfilter wirkungsvoll zur Geltung. Diese modernen Hochleistungstrommelfilter zeichnen sich durch extrem hohe Feststoffdurchsätze, saubere Filterkuchen durch eine intensive Kuchenwäsche mit sehr niedrigem Waschwasserverbrauch, niedrige Restfeuchten und eine vollständige Kuchenabnahme aus. Die Grundlage dafür ist ein gut be- rechnetes und ausgeklügeltes Design des Filterhydrauliksystems. Viele innovative Details erleichtern Betrieb und Wartung dieser modernen Trommelfilter, wie z. B. einzeln austauschbare Filterzellen oder FrameTrak, eine ganz neue Methode der Filtertuchbefestigung für größere Filtereinheiten.

Der Beitrag beschreibt wesentliche Merkmale im Design dieser neuen Trommelfiltergeneration. Weiterhin wird anhand von Fallstudien/Referenzanwendungen aus dem Bereich Petrochemie, Pigmente, Feinmineralien, Zuschlagstoffe etc. gezeigt, wie das moderne Design dieser Filter zu erheblichen Verbesserungen in Betrieb, Wartung und Wirtschaftlichkeit des Trennprozesses beiträgt.

\section{P3.19}

\section{Anwendbarkeit der Lévêque-Analogie bei keramischen Schwämmen}

Dipl.-Ing. B. Dietrich ${ }^{1)}$ (E-Mail: dietrich@kit.edu), Prof. Dr.-Ing. M. Kind ${ }^{1)}$, Prof. Dr.-Ing. H. Martin ${ }^{1)}$

${ }^{1)}$ Institut für Thermische Verfahrenstechnik, Universität Karlsruhe (TH), Karlsruhe Institute of Technology (KIT), Kaiserstraße 12, D-76131 Karlsruhe, Germany

DOI: $10.1002 /$ cite. 200950007

Keramische Schwämme sind hochporöse dreidimensionale Netzstrukturen und werden in der Literatur oft als „open-celled foams“ bezeichnet. Vor mehr als 50 Jahren wurden diese als Metallschmelzen-Filter entwickelt, derzeit wird aber an neuen, für die Verfahrenstechnik potenziell interessanten Anwendungen geforscht. Dies sind z.B.
Solarreceiver, Porenbrenner oder Beheizungssysteme. Zur Auslegung derartiger Apparate ist eine genaue Kenntnis des Wärme- und Impulstransportes notwendig.

Eine wichtige Kenngröße zur Beschreibung des Impulstransportes ist der Druckverlust, welcher durch den Einbau des Schwammes erzeugt wird. Mit Hilfe der experimentell bestimmten Daten war es möglich, eine für alle untersuchten Schwämme gültige Berechnungsvorschrift $\mathrm{zu}$ ermitteln. Der Wärmetransport hingegen wird durch den Wärmeübergangskoeffizienten zwischen Feststoffstruktur und strömendem Fluid charakterisiert. Er wurde mit Hilfe einer instationären Methode bestimmt. 
Die Hypothese ist, die experimentellen Daten des Druckverlustes mit denen des Wärmetransports in Beziehung zu setzen. Aus der Klasse der Wärme- und Impulsanalogien deutet sich für den Fall des thermischen und gleichzeitigem hydrodynamischen Anlaufs die in der Literatur gut bekannte Verallgemeinerte LévêqueGleichung heraus [1], wobei der Wärmetransport von der dritten Wurzel des Reibungsbeiwertes abhängig ist.

Die Abb. zeigt erste Ergebnisse für verschiedene keramische Schwämme. Dabei wurden das Material des Feststoffgerüstes, die Porosität sowie die Porengröße variiert. Es zeigte sich grundsätzlich die vermutete Tendenz und damit die Anwendbarkeit der Verallgemeinerten Lévêque-Gleichung.

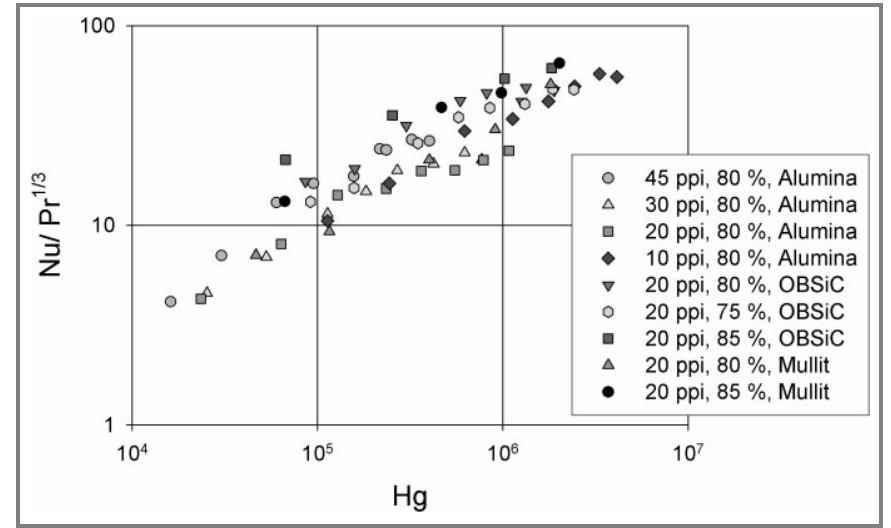

Abbildung. Erste Ergebnisse zur Anwendbarkeit der Verallgemeinerten Lévêque-Gleichung bei Schwämmen.

[1] H. Martin, Chem. Eng. Process. 1996, 35, 301.

\title{
P3.20 \\ Modellierung und Simulation der Strömung im überkritisch betriebenen Monolithreaktor

\author{
Dipl.-Ing H. Häring ${ }^{1)}$ (E-Mail: Hans.Haering@mailbox.tu-dresden.de), Dipl.-Ing. (FH) D. Burgbacher ${ }^{1)}$, Dr.-Ing. T. Bauer ${ }^{1)}$, \\ Prof. Dr.-Ing. habil. W. Klöden ${ }^{1}$, Prof. Dr.-Ing. habil. R. Lange ${ }^{1)}$ \\ 1)Institut für Verfahrenstechnik und Umwelttechnik, TU Dresden, D-01062 Dresden, Germany \\ DOI: $10.1002 /$ cite.200950442
}

Bei der Modellierung des überkritischen Monolithreaktors ist die Betrachtung der Strömung wichtig, denn diese beeinflusst den Stoff- und Wärmetransport in hohem Maß. In der vorliegenden Arbeit werden verschiedene Modelle zur Berechnung der Geschwindigkeitsprofile hinsichtlich Rechenaufwand und Genauigkeit verglichen.

Für die Modellierung wurde als Stoffsystem die Hydrierung von Cyclohexen $\mathrm{zu}$ Cyclohexan in $\mathrm{scCO}_{2}$ untersucht. Die Simulationen wurden für Drücke von 80 - 160 bar, Temperaturen von $40-100^{\circ} \mathrm{C}$ und für Reynoldszahlen von $22-67$ durchgeführt. Es wird ein isothermer und isobarer Betrieb ohne chemische Reaktion mit konstanten Stoffwerten und inkompressibler Strömung betrach- tet. Der Reaktor vereinfacht sich unter der Annahme gleichförmiger Anströmung aller Monolithkanäle $\mathrm{zu}$ einem Einzelkanalmodell.

Die Referenz-Simulation der NSG wurde mit dem Programm COMSOL Multiphysics 3.5 gelöst. Als zweites Modell wurden die NSG durch eine Reihenentwicklung nach Spurk analytisch berechnet. Das einfachste Modell stellt eine empirische Gleichung nach Hartnett und Kostic dar. Als Ergebnis wurde gefunden, dass die Lösung nach Spurk [1] im Kanalzentrum für die stationäre Strömung bei allen Versuchspunkten bis zu $2 \%$ von der numerischen Lösung abweicht. Am Rand des Kanals treten durch den Reihenabbruch Abweichungen bis ca. $10 \%$ auf. Die Korrelation nach Hartnett und Kostic [2] beschreibt die simulierten Werte im Zentrumsbereich mit bis zu $10 \%$ Abweichung, aber in den Kanalecken steigt der Fehler auf bis zu $50 \%$.

Zusammenfassend kann die Korrelation nach Hartnett und Kostic zur Abschätzungen empfohlen werden. Zur genaueren Beschreibung der stationären Strömung sollte das Modell nach Spurk verwendet werden. Soll neben der stationär ausgeprägten Strömung auch der Einlaufbereich betrachtet werden, ist die Lösung der NSG notwendig.

[1] J. H. Spurk, Strömungslehre, 6. Aufl., Springer-Verlag, Berlin 2005.

[2] J. Hartnett, M. Kostic, Adv. Heat Transfer 1989, 19, 247.

\section{Efficient energy transfer by hydrodynamic modification} in laminar flow

\author{
B. Olayiwola1) (E-Mail: b.olayiwola@tu-dortmund.de) \\ ${ }^{1)}$ TU Dortmund, Emil-Figge-Straße 70, D-44221 Dortmund, Germany
}

